\title{
sciendo
}

\section{THE INFLUENCE OF HEMPSEED CAKE ON PRODUCTION TRAITS, METABOLIC PROFILE AND ANTIOXIDANT STATUS OF MERINOLANDSCHAF LAMBS}

\author{
Zvonko Antunović ${ }^{1}$, Željka Klir Šalavardić ${ }^{1 \bullet}$, Zvonimir Steiner ${ }^{1}$, Mislav Đidara ${ }^{1}$, Suzana Ćavar ${ }^{2}$, \\ Mario Ronta ${ }^{1}$, Andrijana Modić Šabić ${ }^{2}$, Valentina Pavić 3 , Josip Novoselec ${ }^{1}$
'Department for Animal Production and Biotechnology, Faculty of Agrobiotechnical Sciences Osijek, Josip Juraj Strossmayer University of Osijek, V. Preloga 1, 31000 Osijek, Croatia ${ }^{2}$ Institute of Public Health of the Osijek-Baranja County, F. Krežme 1, 31000 Osijek, Croatia ${ }^{3}$ Department of Biology, Josip Juraj Strossmayer University of Osijek, Cara Hadrijana 8/A, 31000, Osijek \\ •Corresponding author: zklir@fazos.hr
}

\begin{abstract}
The aim of this study was to investigate the effect of hempseed cake on production traits, metabolic profile and antioxidant status of lambs. The research was conducted on 20 Merinolandschaf lambs of similar live body weight $(24.03 \mathrm{~kg} \pm 2.22$ and $24.06 \pm 1.84 \mathrm{~kg}$ in control and experimental group, respectively). The experiment started after the weaning of lambs at their average age of 70 days. Lambs were divided by gender (50\% male: $50 \%$ female) and then allocated in two groups. The research lasted for 30 days during the fattening period. Lambs in the control group were fed with feed mixtures, where main protein source was soybean meal and extruded soybean (SB), while lambs in the experimental group were fed feed mixtures in which soybean was replaced by hempseed cake (HSC) at $12 \%$ of dry matter. Higher values of daily weight gain $(\mathrm{P}<0.05)$ compared to SB lambs were observed from days 15 to 30 . HSC lambs showed no significant differences in hematology and biochemical blood parameters, except for lower $(\mathrm{P}<0.001)$ urea concentrations on days 15 and 30, as well as higher $(\mathrm{P}<0.05) \mathrm{Mg}$ concentrations in serum on day 30 . Hempseed cake in lambs' diets did not influence $(P>0.05)$ activity of the enzymes like AST, ALT, ALP, GGT and CK, as well as SOD and GPx enzymes, thus not affecting the antioxidant status of lambs' blood. Concentrations of DHA and $\mathbf{n - 3}$ fatty acids were higher $(\mathrm{P}<0.05)$ in $\mathrm{HSC}$ group than in SB group. The present study confirmed that the inclusion of HSC as soybean replacement up to $12 \%$ in the lambs' diet is justified when considering production traits, metabolic profile and antioxidant status of Merinolandschaf lambs.
\end{abstract}

Key words: antioxidant status, hempseed cake, lambs, metabolic profile, production traits

The importance of protein component in a diet depends on its quality and price. Recently, many feedstuffs and by-products of various foods have been used as an alternative to soybean, which is of genetically modified organism (GMO) origin and 
not competitively priced. Pumpkin seed cake, peas, sunflower seed cake (Klir et al., 2017; Antunović et al., 2017, 2018 and 2019), and recently hempseed cake have been used instead of soybean. Hempseed cake is a by-product of oil extraction from hemp seed and it is rich in high-quality storage proteins (Wang and Xiong, 2019). Contemporary hemp varieties have much lower proportion of cannabinoids. In most European countries, the current upper legal limit for cultivation of hemp for fiber and seeds production is $0.2 \%$ THC (D9-tetrahydrocannabinol) on a dry weight basis (Russo and Reggiani, 2013). Karlsson et al. (2010) reported that hempseed cake contains $937 \mathrm{~g} / \mathrm{kg}$ of DM, $344 \mathrm{~g} / \mathrm{kg}$ DM of crude protein, $124 \mathrm{~g} / \mathrm{kg}$ DM of crude fat, 393 $\mathrm{g} / \mathrm{kg}$ DM of NDF (neutral detergent fiber) and $321 \mathrm{~g} / \mathrm{kg}$ DM of ADF (acid detergent fiber). Hemp seeds and cakes are rich in polyunsaturated fatty acids (PUFA) and phytosterols that have been recognized as potential therapeutic agents for cardiac protection. Hemp seeds and hempseed cake contain tocopherols, especially $\gamma$-tocopherol (60.85 and $33.72 \mathrm{mg} / 100 \mathrm{~g} \mathrm{DM}$, respectively), which increase oxidative stability of sheep milk (Mierlita, 2018). Klir et al. (2019) indicated the possibility of using hempseed cake in animal diets without causing major changes in production and possible increase of desirable fatty acids in animal products.

There are numerous studies on the use of hempseed cake as a substitute for soybean in ruminant diets and on its impact on milk quantity and quality, with particular emphasis on the fatty acid profile in cow and sheep milk (Karlsson et al., 2010; Mierlita, 2016, 2018) and in meat (Hessle et al., 2008; Turner et al., 2008, 2012; Karlsson and Martinson, 2011). However, there are not many researches on how the hempseed cake in ruminant feeding affects antioxidant status of ruminants (Mierlita, 2018). Thus, the aim of this study was to investigate the effect of hempseed cake on production characteristics, metabolic profile and antioxidant status of lambs.

\section{Material and methods}

\section{Animals selection, experimental design and diet}

The Bioethics Committee for Research on Animals of the Faculty of Agrobiotechnical Sciences Osijek established that the present research was carried out under the legal provisions according to the Animal Protection Act (OJ 133/06, OJ 37/13 and OJ 125/13). Animal handling practices are in line with the European Union Directive 2010/63/EU about the protection of animals used for the experiment. The research was conducted on 20 Merinolandschaf lambs, after they were weaned at the average age of 70 days. Animals used in this study were maintained in facilities approved by the Croatian Association for Accreditation of Laboratory Animal Care, and in accordance with current regulations and standards issued by the Croatian Ministry of Agriculture. Lambs were of good health and evenly divided by gender $(50 \%$ male and 50\% female) and then further divided into two groups. Feeding of lambs was carried out in a control group with feed mixtures whose main protein source was soybean and by-products of soybean meal (SB), while in the experimental group the soybean and soybean meal were partially replaced by hempseed cake (HSC) in the amount of $12 \%$ DM. Hay and water were offered to lambs ad libitum. Feed mixtures 
were offered to lambs twice per day, while no refusals were observed. Duration of experiment was 30 days.

Feed composition was determined with standard methods (AOAC, 2006) and presented in Table 1. Concentrate mixtures were isoproteic, isolipidic and isoenergetic formulated according to the National Research Council (2007). Crude protein content (CP) of feed samples was determined by the Kjeldahl method, while ether extract (EE) was determined by using the Universal Extractions System B-811 (Buchi, Switzerland). Determination of amylase-treated NDF was determined according to the EN ISO 1647:2006 (European Committee for Standardization, 2006), while acid detergent fiber (ADF) was determined according to the EN ISO 13906 (European Committee for Standardization, 2008). Non-fiber carbohydrates were obtained by the following equation (Sniffen et al., 1992): non-fiber carbohydrates (NFC) $=100-$ $(\% \mathrm{NDF}+\% \mathrm{CP}+\% \mathrm{EE}+\%$ ash $)$. Ash was determined by incinerating the feed samples at $550^{\circ} \mathrm{C}$ during 6 hours. Weighing and measuring of the exterior characteristics of lambs (height of withers, body length, chest depth and width, circumference of body chest and of shin bone, ham length and circumference), as well as body condition score were carried out on days 1, 15 and 30. Body condition score of lambs was determined according to Russel (1991).

Table 1. Ingredients of feed mixtures, chemical composition, and fatty acid concentration of feed mixtures and hay

\begin{tabular}{l|c|c|c}
\hline \multirow{2}{*}{ Component $(\mathrm{g} / \mathrm{kg}$ as fed) } & \multicolumn{3}{c}{ Groups } \\
\cline { 2 - 4 } \multicolumn{1}{c|}{1} & SB & HSC & Meadow hay \\
\hline \multicolumn{1}{c}{ Ingredient proportion } & & 3 & 4 \\
corn grain & 500 & 500 & - \\
barley grain & 130 & 130 & - \\
wheat flour & 100 & 100 & - \\
soybean meal & 100 & 70 & - \\
extruded soybean & 140 & 50 & - \\
hempseed cake & - & 120 & - \\
mineral premix* & 30 & 30 & - \\
Chemical composition (g/kg dry matter) & & & \\
dry matter & 926.05 & 928.40 & 899.50 \\
crude protein & 162.50 & 160.80 & 141.90 \\
ether extract & 57.40 & 50.50 & 16.20 \\
crude fiber & 33.70 & 53.10 & 125.20 \\
ash & 57.70 & 55.50 & 62.20 \\
neutral detergent fiber & 241 & 388.2 & 504.7 \\
acid detergent fiber & 4.64 & 6.27 & 21.49 \\
non-fibrous carbohydrates & 48.14 & 34.50 & 27.50 \\
net energy for growth and fattening (MJ/kg) & 8.19 & 8.15 & 4.45 \\
\hline
\end{tabular}


Table 1 - contd.

\begin{tabular}{lr|r|r}
\hline \multicolumn{1}{c|}{1} & 2 & 3 & 4 \\
\hline Fatty acids (g/100 g FAME) & & & \\
C16:0 & 13.79 & 12.68 & 31.76 \\
C16:1 c9 & 0.13 & 0.15 & 2.98 \\
C17:0 & 0.10 & 0.09 & 0.88 \\
C18:0 & 2.98 & 2.53 & 3.85 \\
C18:1 c9 (OA) & 23.48 & 21.31 & 0.47 \\
C18:2 c9,12 (LA) & 53.85 & 54.23 & 18.59 \\
C18:3 c9c12 c15 (ALA) & 0.31 & 5.71 & 0.00 \\
saturated fatty acids ( $\sum$ SFA) & 22.04 & 28.92 & 67.72 \\
unsaturated fatty acids ( $\sum$ UFA) & 77.96 & 71.08 & 32.28 \\
monounsaturated fatty acids ( $\sum$ MUFA) & 23.64 & 1.55 & 10.18 \\
polyunsaturated fatty acids ( $\sum$ PUFA) & 54.33 & 69.53 & 22.10 \\
\hline
\end{tabular}

SB: soybean group; HSC: hempseed cake group; *Mineral premix: $8 \% \mathrm{Ca}, 5 \% \mathrm{P}, 9.5 \% \mathrm{Na}, 2.00 \% \mathrm{Mg}, 400$ $000 \mathrm{IU}$ vitamin A, $40000 \mathrm{IU}$ vitamin D, $500 \mathrm{mg}$ vitamin E, $4000 \mathrm{mg} \mathrm{Zn,} 2000 \mathrm{mg} \mathrm{Mn}, 60 \mathrm{mg} \mathrm{I}, 10 \mathrm{mg} \mathrm{Co}, 50$ mg Se.

After slaughtering and bleeding of lambs, lamb skin was peeled off the carcass, and abdominal (forestomach, stomach, spleen, intestine and liver) and thoracic (trachea with the lungs and heart) cavity organs were then removed. Afterwards, samples of muscle ( $m$. semimembranosus) and liver were taken. The following parts of lamb carcass were measured: the length of the carcass (carcass length 1 - os pubis to atlas; carcass length 2 - os pubis to first rib; and carcass length 3 - os pubis to last rib), the circumference of the carcass (at scapula) and the length of the hind legs (tuber calcanei to tubercle ossis ischia) and hind leg circumference (the widest part) by using the method developed by Fischer and De Boer (1994); and weight of internal organs (lungs, heart, liver, spleen), weight of pre-stomach and intestines, and weight of lower legs and skin. Afterwards, dressing percentage was calculated as: $100 \times$ (carcass weight/live body weight). Values of $\mathrm{pH}_{1}$ were taken 45 min post mortem and $\mathrm{pH}_{2} 24 \mathrm{~h}$ after slaughter and cooling. The $\mathrm{pH}$ was measured with a handheld Mettler Toledo (Greifensee, Switzerland) contact $\mathrm{pH}$ meter with piercing type electrode. Values of carcass color were determined in $m$. semimembranosus $45 \mathrm{~min}$ after slaughtering and measured according to the CIE L* a* b* system (CIE, 1976).

\section{Blood samples collection and analysis}

Blood was taken 2 hours after morning feeding from the jugular vein $(10 \mathrm{ml})$ on days 0,15 and 30 of experiment and put into serum vacutainer tubes and vacutainer tubes containing ethylenediamine tetra-acetic acid. Hematological parameters, leukocytes (WBC), erythrocytes (RBC) and thrombocytes (PLT counts), as well as content of hemoglobin (HGB), hematocrit (HCT), mean corpuscular volume (MCV), the average hemoglobin content in erythrocytes $(\mathrm{MCH})$ and mean hemoglobin con- 
centration in erythrocytes (MCHC) were determined in whole blood of lambs. The hematological analysis was performed with automated 3-part differential hematology analyzer Sysmex PocH-100Iv (Sysmex Europe GmbH, Hamburg, Germany).

Blood serum was separated by centrifugation at $1609.92 \mathrm{~g}$ within 10 minutes. Concentrations of $\mathrm{Ca}$, P-inorganic, $\mathrm{Mg}, \mathrm{Fe}$, biochemical parameters: urea, glucose, cholesterol, high-density lipoprotein (HDL), low-density lipoprotein (LDL), triglycerides, total protein, albumin, globulin, non-esterified fatty acids (NEFA), betahydroxybutyrate (BHBA) and enzyme activities: alanine aminotransferase (ALT), aspartate aminotransferase (AST), alkaline phosphatase (ALP), creatine kinase (CK) and $\gamma$-glutamyl transferase (GGT) were determined in serum. The concentration of BHBA was measured with the colorimetric method (RANBUT, Laboratories Ltd., Crumlin, UK). The activity of glutathione peroxidase (GPx) in serum was determined by Ransel ${ }^{\circledR}$ kits (Randox, UK) and the activity of total superoxide dismutase (SOD) with Ransod ${ }^{\circledR}$ kits (Randox, UK). All parameters determined in serum were analysed with Olympus AU400 analyser (Tokyo, Japan).

\section{Measurements of lipid peroxidation and antioxidative activity of meat and liver}

The thiobarbituric acid reactive substances (TBARS) were measured according to standard methodology (Liu et al., 2010) with slight modifications. The absorbances were measured at $532 \mathrm{~nm}$ and $600 \mathrm{~nm}$ by using a spectrophotometer (Lambda 25, Perkin Elmer). The malondialdehyde (MDA) concentration was calculated by using the molar extinction coefficient of $\operatorname{MDA}\left(156,000 \mathrm{M}^{-1} \mathrm{~cm}^{-1}\right)$. The results were reported as mg MDA per $\mathrm{kg}$ meat sample (mg MDA $/ \mathrm{kg}$ meat).

The 2,2-diphenyl-1-picrylhydrazyl (DPPH) of the muscle and liver samples was measured according to the method of Qwele et al. (2013). The absorbance of the solution was measured at $517 \mathrm{~nm}$ by using a spectrophotometer (Lambda 25, Perkin Elmer). The scavenging activity of meat sample against DPPH radical was expressed as inhibiting percent and calculated as:

$\%$ DPPH radical scavenging activity $=1-($ A sample - A blank $) /($ A control sample) $\times 100$

\section{Fatty acid analysis}

Fatty acid methyl esters (FAME) were extracted from feed and m. semimembranosis. Reference for FAME consisted of 37 fatty acid methyl esters (purity $\geq 99 \%$ ) purchased from Restek (Pennsylvania, USA). Fatty acid methyl esters from fat were prepared according to International Standard HRN ISO 15884:2002 (Croatian Standards Institute, 2003). Approximately $100 \mathrm{mg}$ of the prepared test sample (fat) was dissolved in $5 \mathrm{ml}$ of $\mathrm{n}$-heptane in a test tube. Then $0.2 \mathrm{ml}$ of the transesterification reagent $(\mathrm{KOH}$, methanolic solution) was added and vortexed (Vortexer, Grant Instruments Ltd, England) for $1 \mathrm{~min}$. After reaction time of $5 \mathrm{~min}, 0.5 \mathrm{~g}$ of solid sodium hydrogen sulphate was added and mixed well. The test tubes were centrifuged (Hettich Zentrifugen, Tuttlingen, Germany) for $3 \mathrm{~min}$, at $2000 \mathrm{rpm}$ and at a room temperature. An aliquot of ester solution (clear supernatant) was taken for chromatographic analysis. 
Fatty acid methyl esters from meat and feed were prepared according to the HRN EN ISO 12966-2:2017 (Croatian Standards Institute, 2017). Test sample in the amount of 100-200 mg was prepared with addition of $4 \mathrm{ml}$ of methanolic sodium hydroxide $(0.5 \mathrm{M})$ in $50 \mathrm{ml}$ flask with $2-3$ boiling chips. Flask was boiled under reflux for $20 \mathrm{~min}$ and then $5 \mathrm{ml}$ of methanolic boron trifluoride solution was added through the top of the condenser and boiled for 4 min. Fatty acids were then extracted with $5 \mathrm{ml}$ of n-heptane and boiled for additional $2 \mathrm{~min}$. After the flask cooled down, saturated aqueous sodium chloride solution was added. Few millilitres of upper n-heptane layer were transferred to test tube that contained anhydrous sodium sulfate. There was $1 \mathrm{ml}$ of aliquot taken for chromatographic analysis.

Analysis of the fatty acids was performed by using Agilent 7890B (Agilent, Santa Clara, USA) gas chromatograph with flame ionization detector. Carrier gas was $\mathrm{N}_{2}$ in split mode with split ratio 20:1. Inlet pressure was 35.81 psi with temperature $225^{\circ} \mathrm{C}$ and $28.2 \mathrm{ml} / \mathrm{min}$ total flow. Column flow was $1.2 \mathrm{ml} / \mathrm{min}$, and column used for separation of fatty acids was fuse silica-capillary column Rt-2560, biscyanopropyl polysiloxane-not bonded (100 $\mathrm{m} \times 250 \mu \mathrm{m}$ i.d., $0.2 \mu \mathrm{m}$ film thickness) (Restek, Pennsylvania, USA). The oven temperature was maintained at $100^{\circ} \mathrm{C}$ for $4 \mathrm{~min}$, then heated to $240^{\circ} \mathrm{C}$ for $11 \mathrm{~min}$ with ramp of $3^{\circ} / \mathrm{min}$. Injection volume of sample was $1 \mu \mathrm{l}$. Detector temperature was $250^{\circ} \mathrm{C}$ and make flow $\left(\mathrm{N}_{2}\right)$ $45 \mathrm{ml} / \mathrm{min}$.

\section{Statistical analysis}

The distribution of data was verified by the Shapiro-Wilk test (PROC UNIVARIATE). Results were expressed as mean values and standard error of mean estimated by MEANS procedure. Effects of treatment (SB-group; HSC-group) were analysed by GLM procedure. Means were compared by the Tukey's test and differences between the groups were declared significant at $\mathrm{P}<0.05$, while $\mathrm{P} \leq 0.10$ were considered as data trends. Research results were processed by SAS (SAS Inst. Inc., Cary NC) software.

\section{Results}

Analysis of exterior and production traits of HSC and SB group of lambs showed almost no significant differences, although HSC group had higher values of daily weight gain $(\mathrm{P}<0.05)$ compared to $\mathrm{SB}$ lambs observed from days 15 to 30 (Tables 2 and 3$)$.

When compared to the SB group, metabolic profile of HSC lamb blood revealed no significant differences except for lower $(\mathrm{P}<0.05) \mathrm{MCHC}$ content in whole blood on day 15 and lower $(\mathrm{P}<0.001)$ urea concentrations on days 15 and 30 , as well as higher $\mathrm{Mg}$ concentrations in blood on day 30 (Tables 4, 5 and 6). Hempseed cake in lambs' diets did not influence activity of the enzymes like AST, GGT, ALT, ALP, and CK, as well as SOD and GPx enzymes compared to SB (Table 7). 
Table 2. Production traits and average daily intake and feed conversion of lambs

\begin{tabular}{l|r|c|c|c|c}
\hline \multirow{2}{*}{\multicolumn{1}{c}{ Indicator }} & \multirow{2}{*}{ Days } & \multicolumn{2}{c|}{ Group (mean) } & \multirow{2}{*}{ SEM } & \multirow{2}{*}{ P-value } \\
\cline { 3 - 4 } & & SB & HSC & & \\
\hline Body weight (kg) & 1 & 24.03 & 24.06 & 0.424 & 0.967 \\
& 15 & 28.36 & 29.57 & 0.709 & 0.407 \\
Daily weight gain (kg/day) & 30 & 32.21 & 34.05 & 0.817 & 0.270 \\
& $1-15$ & 0.331 & 0.367 & 0.035 & 0.625 \\
& $15-30$ & 0.223 & 0.298 & 0.017 & 0.022 \\
Average daily intake (kg/day) & $1-30$ & 0.273 & 0.333 & 0.021 & 0.202 \\
& $1-15$ & 1.07 & 0.86 & - & - \\
Feed conversion $(\mathrm{kg} / \mathrm{kg})$ & $15-30$ & 0.90 & 0.93 & - & - \\
& $1-30$ & 0.99 & 0.89 & - & - \\
& $1-15$ & 3.24 & 2.33 & - & - \\
\hline
\end{tabular}

Mean: mean value; SEM: standard error of mean; SB: soybean group; HSC: hempseed cake group.

Table 3. Exterior characteristics and body condition score of lambs

\begin{tabular}{|c|c|c|c|c|c|}
\hline \multirow{2}{*}{ Indicator $(\mathrm{cm})$} & \multirow{2}{*}{ Day } & \multicolumn{2}{|c|}{ Group (mean) } & \multirow{2}{*}{ SEM } & \multirow{2}{*}{ P-value } \\
\hline & & SB & $\mathrm{HSC}$ & & \\
\hline \multirow[t]{3}{*}{ Height of withers } & 1 & 54.14 & 52.77 & 0.672 & 0.321 \\
\hline & 15 & 55.55 & 56.00 & 0.665 & 0.742 \\
\hline & 30 & 57.20 & 58.45 & 0.545 & 0.452 \\
\hline \multirow[t]{3}{*}{ Body length } & 1 & 54.64 & 54.73 & 0.632 & 0.945 \\
\hline & 15 & 59.26 & 58.86 & 0.567 & 0.734 \\
\hline & 30 & 59.85 & 63.36 & 1.050 & 0.095 \\
\hline \multirow[t]{3}{*}{ Chest depth } & 1 & 19.91 & 19.95 & 0.155 & 0.888 \\
\hline & 15 & 20.64 & 21.35 & 0.247 & 0.156 \\
\hline & 30 & 21.45 & 21.92 & 0.205 & 0.236 \\
\hline \multirow[t]{3}{*}{ Chest width } & 1 & 14.36 & 14.14 & 0.122 & 0.364 \\
\hline & 15 & 16.13 & 15.08 & 0.376 & 0.170 \\
\hline & 30 & 16.48 & 15.61 & 0.259 & 0.094 \\
\hline \multirow{3}{*}{$\begin{array}{l}\text { Circumference } \\
\text { of body at chest }\end{array}$} & 1 & 64.18 & 63.82 & 0.399 & 0.660 \\
\hline & 15 & 68.45 & 68.55 & 0.573 & 0.939 \\
\hline & 30 & 73.70 & 74.27 & 0.704 & 0.695 \\
\hline \multirow[t]{3}{*}{ Circumference of shin bone } & 1 & 7.65 & 7.71 & 0.076 & 0.738 \\
\hline & 15 & 7.84 & 7.85 & 0.084 & 0.958 \\
\hline & 30 & 8.47 & 8.70 & 0.110 & 0.312 \\
\hline \multirow[t]{3}{*}{ Ham length } & 1 & 27.86 & 27.64 & 0.355 & 0.758 \\
\hline & 15 & 28.73 & 28.27 & 0.501 & 0.663 \\
\hline & 30 & 29.80 & 30.73 & 0.294 & 0.117 \\
\hline \multirow[t]{3}{*}{ Ham circumference } & 1 & 37.73 & 37.59 & 0.648 & 0.919 \\
\hline & 15 & 38.32 & 40.32 & 0.390 & 0.051 \\
\hline & 30 & 44.70 & 46.27 & 0.615 & 0.209 \\
\hline \multirow[t]{3}{*}{ Body condition score } & 1 & 3.44 & 3.53 & 0.069 & 0.485 \\
\hline & 15 & 3.43 & 3.71 & 0.080 & 0.079 \\
\hline & 30 & 3.75 & 4.03 & 0.087 & 0.105 \\
\hline
\end{tabular}

Mean: mean value; SEM: standard error of mean; SB: soybean group; HSC: hempseed cake group. 
Table 4. Hematological parameters of lambs

\begin{tabular}{|c|c|c|c|c|c|}
\hline \multirow{2}{*}{ Indicator } & \multirow{2}{*}{ Day } & \multicolumn{2}{|c|}{ Group (mean) } & \multirow{2}{*}{ SEM } & \multirow{2}{*}{ P-value } \\
\hline & & SB & HSC & & \\
\hline \multirow[t]{3}{*}{ White blood cells $\left(\times 10^{9} / \mathrm{L}\right.$ blood $)$} & 1 & 10.25 & 12.00 & 0.795 & 0.283 \\
\hline & 15 & 9.82 & 9.76 & 0.799 & 0.317 \\
\hline & 30 & 8.60 & 9.17 & 0.502 & 0.585 \\
\hline \multirow[t]{3}{*}{ Red blood cells $\left(\times 10^{12} / \mathrm{L}\right.$ blood $)$} & 1 & 9.69 & 10.03 & 0.210 & 0.428 \\
\hline & 15 & 11.06 & 9.76 & 0.409 & 0.115 \\
\hline & 30 & 8.22 & 8.55 & 0.417 & 0.706 \\
\hline \multirow[t]{3}{*}{ Hemoglobin (g/L blood) } & 1 & 124.36 & 119.63 & 3.488 & 0.511 \\
\hline & 15 & 135.18 & 107.91 & 7.099 & 0.052 \\
\hline & 30 & 101.40 & 96.73 & 3.984 & 0.571 \\
\hline \multirow[t]{3}{*}{ Hematocrit (g/L blood) } & 1 & 0.37 & 0.39 & 0.010 & 0.279 \\
\hline & 15 & 0.43 & 0.38 & 0.018 & 0.277 \\
\hline & 30 & 0.34 & 0.35 & 0.011 & 0.662 \\
\hline \multirow[t]{3}{*}{ Mean corpuscular volume (fL) } & 1 & 37.95 & 38.97 & 0.347 & 0.146 \\
\hline & 15 & 38.57 & 39.36 & 0.355 & 0.276 \\
\hline & 30 & 43.56 & 42.15 & 1.742 & 0.700 \\
\hline \multirow[t]{3}{*}{ Mean corpuscular hemoglobin (pg) } & 1 & 12.55 & 11.98 & 0.246 & 0.254 \\
\hline & 15 & 12.19 & 11.09 & 0.268 & 0.051 \\
\hline & 30 & 12.74 & 11.56 & 0.452 & 0.201 \\
\hline \multirow{3}{*}{$\begin{array}{l}\text { Mean corpuscular hemoglobin } \\
\text { concentration }(\mathrm{g} / \mathrm{L} \text { blood })\end{array}$} & 1 & 332.27 & 298.73 & 9.106 & 0.064 \\
\hline & 15 & $316.91 \mathrm{a}$ & $282.27 \mathrm{~b}$ & 7.640 & 0.020 \\
\hline & 30 & 295.30 & 275.27 & 6.891 & 0.151 \\
\hline \multirow[t]{3}{*}{ Platelet blood count $\left(\times 10^{9} / \mathrm{L}\right.$ blood $)$} & 1 & 750.18 & 597.55 & 45.777 & 0.096 \\
\hline & 15 & 750.45 & 759.82 & 41.331 & 0.913 \\
\hline & 30 & 548.30 & 558.45 & 45.941 & 0.916 \\
\hline
\end{tabular}

Mean: mean value; SEM: standard error of mean; SB: soybean group; HSC: hempseed cake group; Values in each row with different letters show significant difference $(\mathrm{P}<0.05)$.

Table 5. Mineral concentration in blood serum of lambs

\begin{tabular}{l|r|c|c|c|c}
\hline \multirow{2}{*}{ Indicator $(\mathrm{mmol} / \mathrm{L})$} & \multirow{2}{*}{ Day } & \multicolumn{2}{|c|}{ Group (mean) } & \multirow{2}{*}{ SEM } & \multirow{2}{*}{ P-value } \\
\cline { 3 - 4 } & & $\mathrm{SB}$ & HSC & & \\
\hline $\mathrm{Mg}$ & 1 & 0.72 & 0.73 & 0.027 & 0.885 \\
& 15 & 0.83 & 1.00 & 0.048 & 0.081 \\
& 30 & $0.82 \mathrm{a}$ & $1.06 \mathrm{~b}$ & 0.051 & 0.014 \\
$\mathrm{Ca}$ & 1 & 2.38 & 2.38 & 0.031 & 0.978 \\
& 15 & 2.56 & 2.47 & 0.035 & 0.170 \\
& 30 & 2.73 & 2.71 & 0.027 & 0.766 \\
$\mathrm{Fe}(\mu \mathrm{mol} / \mathrm{L})$ & 1 & 24.06 & 29.75 & 1.696 & 0.095 \\
& 15 & 32.61 & 36.00 & 2.115 & 0.436 \\
& 30 & 43.34 & 35.77 & 2.818 & 0.187 \\
$\mathrm{P}$ & 1 & 3.68 & 3.71 & 0.121 & 0.971 \\
& 15 & 3.14 & 4.39 & 0.196 & 0.051 \\
& 30 & 3.94 & 3.52 & 0.139 & 0.076 \\
\hline
\end{tabular}

Mean: mean value; SEM: standard error of mean; SB: soybean group; HSC: hempseed cake group; Mg: magnesium; Ca: calcium; Fe: iron; P: phosphorus; values in each row with different letters show significant difference $(\mathrm{P}<0.05)$. 
Table 6. Biochemical parameters in blood serum of lambs

\begin{tabular}{|c|c|c|c|c|c|}
\hline \multirow{2}{*}{ Blood metabolites } & \multirow{2}{*}{ Day } & \multicolumn{2}{|c|}{ Group (mean) } & \multirow{2}{*}{ SEM } & \multirow{2}{*}{ P-value } \\
\hline & & SB & HSC & & \\
\hline \multirow[t]{3}{*}{ Glucose $(\mathrm{mmol} / \mathrm{L})$} & 1 & 4.39 & 4.33 & 0.099 & 0.756 \\
\hline & 15 & 5.15 & 5.28 & 0.129 & 0.638 \\
\hline & 30 & 4.61 & 4.43 & 0.108 & 0.412 \\
\hline \multirow[t]{3}{*}{ Urea $(\mathrm{mmol} / \mathrm{L})$} & 1 & 3.47 & 3.52 & 0.208 & 0.909 \\
\hline & 15 & $8.12 \mathrm{a}$ & $5.51 \mathrm{~b}$ & 0.419 & $<0.001$ \\
\hline & 30 & $10.62 \mathrm{a}$ & $7.97 \mathrm{~b}$ & 0.440 & $<0.001$ \\
\hline \multirow[t]{3}{*}{ Total protein $(\mathrm{g} / \mathrm{L})$} & 1 & 59.27 & 61.37 & 0.663 & 0.246 \\
\hline & 15 & 64.27 & 61.42 & 1.239 & 0.259 \\
\hline & 30 & 67.07 & 67.31 & 0.774 & 0.882 \\
\hline \multirow[t]{3}{*}{ Albumin $(\mathrm{g} / \mathrm{L})$} & 1 & 27.84 & 29.71 & 0.410 & 0.051 \\
\hline & 15 & 29.43 & 29.29 & 0.319 & 0.837 \\
\hline & 30 & 31.04 & 31.44 & 0.343 & 0.578 \\
\hline \multirow[t]{3}{*}{ Globulin (g/L) } & 1 & 31.96 & 31.66 & 0.664 & 0.828 \\
\hline & 15 & 34.85 & 32.13 & 1.287 & 0.302 \\
\hline & 30 & 36.03 & 35.87 & 0.694 & 0.913 \\
\hline \multirow[t]{3}{*}{ Cholesterol (mmol/L) } & 1 & 2.05 & 2.22 & 0.156 & 0.612 \\
\hline & 15 & 1.42 & 1.39 & 0.077 & 0.580 \\
\hline & 30 & 1.46 & 1.38 & 0.064 & 0.556 \\
\hline \multirow[t]{3}{*}{ Triglycerides (mmol/L) } & 1 & 0.25 & 0.23 & 0.016 & 0.628 \\
\hline & 15 & 0.30 & 0.29 & 0.025 & 0.786 \\
\hline & 30 & 0.34 & 0.31 & 0.018 & 0.396 \\
\hline \multirow[t]{3}{*}{ HDL (mmol/L) } & 1 & 1.00 & 1.13 & 0.057 & 0.240 \\
\hline & 15 & 0.73 & 0.73 & 0.026 & 0.960 \\
\hline & 30 & 0.83 & 0.71 & 0.056 & 0.118 \\
\hline \multirow[t]{3}{*}{ LDL (mmol/L) } & 1 & 0.98 & 0.98 & 0.102 & 0.997 \\
\hline & 15 & 0.55 & 0.47 & 0.055 & 0.217 \\
\hline & 30 & 0.54 & 0.45 & 0.048 & 0.453 \\
\hline \multirow[t]{3}{*}{ NEFA (mmol/L) } & 1 & 0.50 & 0.36 & 0.116 & 0.556 \\
\hline & 15 & 0.36 & 0.28 & 0.098 & 0.315 \\
\hline & 30 & 0.27 & 0.24 & 0.083 & 0.280 \\
\hline \multirow[t]{3}{*}{$\mathrm{BHB}(\mathrm{mmol} / \mathrm{L})$} & 1 & 0.33 & 0.39 & 0.025 & 0.211 \\
\hline & 15 & 0.29 & 0.34 & 0.021 & 0.300 \\
\hline & 30 & 0.27 & 0.24 & 0.014 & 0.201 \\
\hline
\end{tabular}

Mean: mean value; SEM: standard error of mean; SB: soybean group; HSC: hempseed cake group; HDL: high-density lipoprotein; LDL: low-density lipoprotein; NEFA: non-esterified fatty acids; BHB: $\beta$-hydroxybutyric acid; Values in each row with different letters show significant difference $(\mathrm{P}<0.05)$.

Table 7. Activity of enzymes in blood serum of lambs

\begin{tabular}{c|c|c|c|c|c}
\hline \multirow{2}{*}{ Enzymes } & \multirow{2}{*}{ Day } & \multicolumn{2}{|c|}{ Group (mean) } & \multirow{2}{*}{ SEM } & \multirow{2}{*}{ P-value } \\
\cline { 3 - 4 } & & SB & HSC & & \\
\hline \multicolumn{1}{c|}{1} & 2 & 3 & 4 & 5 & 6 \\
\hline Aspartate transaminase & 1 & 103.91 & 104.94 & 3.559 & 0.889 \\
$($ AST, U/L) & 15 & 140.67 & 104.42 & 14.402 & 0.216 \\
& 30 & 161.42 & 111.51 & 24.011 & 0.311 \\
\hline
\end{tabular}


Table 7 - contd.

\begin{tabular}{lr|r|r|r|c}
\hline \multicolumn{1}{c|}{1} & 2 & 3 & 4 & 5 & 6 \\
\hline Alanine aminotransferase & 1 & 10.90 & 9.82 & 0.496 & 0.286 \\
(ALT, U/L) & 15 & 11.99 & 11.76 & 0.526 & 0.835 \\
& 30 & 11.77 & 14.05 & 0.906 & 0.218 \\
Alkaline phosphatase & 1 & 424.46 & 407.28 & 30.628 & 0.787 \\
(ALP, U/L) & 15 & 454.29 & 580.85 & 44.446 & 0.159 \\
& 30 & 428.82 & 513.09 & 33.064 & 0.211 \\
Gamma-glutamyl trans- & 1 & 69.92 & 86.46 & 4.692 & 0.077 \\
ferase (GGT, U/L) & 15 & 113.98 & 81.03 & 21.098 & 0.448 \\
& 30 & 82.44 & 66.40 & 5.788 & 0.172 \\
Creatine kinase (CK, U/L) & 1 & 150.46 & 162.27 & 9.626 & 0.553 \\
& 15 & 181.91 & 151.09 & 13.303 & 0.256 \\
& 30 & 146.60 & 173.55 & 8.519 & 0.116 \\
Superoxide dismutase & 1 & 0.39 & 0.49 & 0.039 & 0.202 \\
(SOD, U/mL) & 15 & 0.58 & 0.70 & 0.048 & 0.216 \\
& 30 & 0.52 & 0.66 & 0.044 & 0.115 \\
Glutathione peroxidase & 1 & 656.68 & 774.68 & 53.331 & 0.279 \\
(GPx, U/L) & 15 & 920.11 & 950.09 & 109.416 & 0.899 \\
& 30 & 957.06 & 1081.88 & 40.989 & 0.131 \\
\hline
\end{tabular}

Mean: mean value; SEM: standard error of mean; SB: soybean group; HSC: hempseed cake group.

Table 8. Carcass traits, colour indices, and $\mathrm{pH}$ values of musculus semimebranosus of lambs

\begin{tabular}{|c|c|c|c|c|}
\hline \multirow[t]{2}{*}{ Parameter } & \multicolumn{2}{|c|}{ Group (mean) } & \multirow{2}{*}{ SEM } & \multirow{2}{*}{ P-value } \\
\hline & SB & HSC & & \\
\hline Slaughter weight $(\mathrm{kg})$ & 32.21 & 34.11 & 0.858 & 0.279 \\
\hline Carcass length $1(\mathrm{~cm})$ & 76.15 & 74.80 & 0.843 & 0.438 \\
\hline Carcass length $2(\mathrm{~cm})$ & 31.02 & 29.75 & 0.511 & 0.545 \\
\hline Carcass length $3(\mathrm{~cm})$ & 51.80 & 52.90 & 0.878 & 0.212 \\
\hline Hind leg circumference $(\mathrm{cm})$ & 39.15 & 40.05 & 0.523 & 0.404 \\
\hline Hind leg length $(\mathrm{cm})$ & 34.60 & 35.10 & 0.425 & 0.051 \\
\hline Hot carcass weight $(\mathrm{kg})$ & 17.34 & 17.25 & 0.436 & 0.927 \\
\hline Dressing $(\%)$ & $53.85 \mathrm{a}$ & $50.68 \mathrm{~b}$ & 0.728 & 0.025 \\
\hline Organs weight* (kg) & 1.41 & 1.36 & 0.049 & 0.623 \\
\hline Forestomach and intestines $(\mathrm{kg})$ & 7.25 & 7.01 & 0.267 & 0.676 \\
\hline Skin and lower legs $(\mathrm{kg})$ & 4.35 & 4.02 & 0.131 & 0.226 \\
\hline Circumference of trunk at chest $(\mathrm{cm})$ & 64.50 & 65.45 & 0.448 & 0.233 \\
\hline Circumference of trunk at hind leg $(\mathrm{cm})$ & 52.50 & 54.15 & 0.678 & 0.301 \\
\hline $\mathrm{pH}_{1}$ values & 6.64 & 6.69 & 0.031 & 0.410 \\
\hline $\mathrm{pH}_{2}$ values & 5.67 & 5.70 & 0.014 & 0.405 \\
\hline $\mathrm{L}^{*}$ & 43.81 & 40.68 & 1.467 & 0.299 \\
\hline$a^{*}$ & 18.70 & 19.17 & 0.196 & 0.232 \\
\hline$b^{*}$ & 1.91 & 2.19 & 0.143 & 0.337 \\
\hline
\end{tabular}

Mean: mean value; SEM: standard error of mean; SB: soybean group; HSC: hempseed cake group; Carcass length 1: os pubis - atlas, carcass length 2: os pubis - first rib; carcass length 3: os pubis - last rib; *weight of lungs, heart, liver and spleen; $\mathrm{pH}_{1}: 45$ min post mortem, $\mathrm{pH}_{2}: 24 \mathrm{~h}$ post mortem; $\mathrm{SD}$ - standard deviation, SEM standard error of mean; Values in each row with different letters show significant difference $(\mathrm{P}<0.05)$. 
Table 9. Fatty acids concentrations in musculus semimembranosus of lambs

\begin{tabular}{|c|c|c|c|c|}
\hline \multirow{2}{*}{ Fatty acids (g/100 g total fatty acids) } & \multicolumn{2}{|c|}{ Group (mean) } & \multirow{2}{*}{ SEM } & \multirow{2}{*}{ P-value } \\
\hline & SB & HSC & & \\
\hline $\mathrm{C} 4: 0$ & 0.02 & 0.03 & 0.003 & 0.071 \\
\hline C6:0 & 0.01 & 0.01 & 0.001 & 0.764 \\
\hline C8:0 & 0.01 & 0.01 & 0.001 & 0.350 \\
\hline C10:0 & 0.15 & 0.15 & 0.017 & 0.961 \\
\hline $\mathrm{C} 11: 0$ & 0.05 & 0.01 & 0.025 & 0.347 \\
\hline C12:0 & 0.28 & 0.50 & 0.060 & 0.068 \\
\hline C13:0 & 0.03 & 0.03 & 0.003 & 0.459 \\
\hline $\mathrm{C} 14: 0$ & 3.63 & 4.91 & 0.529 & 0.238 \\
\hline C14:1 (cis-9) & 0.12 & 0.19 & 0.023 & 0.159 \\
\hline $\mathrm{C} 15: 0$ & 0.60 & 0.65 & 0.049 & 0.638 \\
\hline C15:1 (cis-10) & 0.01 & 0.02 & 0.002 & 0.056 \\
\hline $\mathrm{C} 16: 0$ & 23.19 & 22.78 & 1.948 & 0.713 \\
\hline C16:1 (cis-9) & 1.49 & 0.79 & 0.209 & 0.052 \\
\hline $\mathrm{C} 17: 0$ & 1.57 & 1.46 & 0.090 & 0.555 \\
\hline C17:1 (cis-10) & 0.73 & 0.58 & 0.068 & 0.294 \\
\hline C18:0 & 15.42 & 12.09 & 1.455 & 0.265 \\
\hline C18:1 (trans-9) & 3.16 & 6.75 & 2.005 & 0.386 \\
\hline C18:1 (cis-9) (OA) & 37.93 & 36.66 & 2.699 & 0.822 \\
\hline C18:2 (trans-9,12) & 0.06 & 0.18 & 0.039 & 0.124 \\
\hline C18:2 (cis-9,12) n6 (LA) & 6.79 & 11.00 & 1.205 & 0.079 \\
\hline C20:0 & 0.15 & 0.14 & 0.008 & 0.631 \\
\hline C18:3 (cis-6,9,12) & 0.15 & 0.16 & 0.018 & 0.834 \\
\hline C20:1 (cis-11) & 0.14 & 0.17 & 0.010 & 0.149 \\
\hline C18:3 (cis-9,12,15) n3 (ALA) & 0.78 & 1.02 & 0.068 & 0.083 \\
\hline $\mathrm{C} 21: 0$ & 0.56 & 0.38 & 0.072 & 0.218 \\
\hline C20:2 (cis- 11,14$)$ & 0.11 & 0.16 & 0.020 & 0.215 \\
\hline $\mathrm{C} 22: 0$ & 0.07 & 0.13 & 0.018 & 0.142 \\
\hline C20:3 (cis-8,11,14) & 0.17 & 0.28 & 0.040 & 0.178 \\
\hline C22:1 (cis-13) & 0.06 & 0.07 & 0.014 & 0.553 \\
\hline C20:3 (cis-11,14,17) & 0.03 & 0.05 & 0.005 & 0.214 \\
\hline C20:4 (cis-5,8,11,14) n6 (AA) & 0.07 & 0.10 & 0.012 & 0.154 \\
\hline $\mathrm{C} 23: 0$ & 1.89 & 2.20 & 0.345 & 0.663 \\
\hline $\mathrm{C} 22: 2$ (cis-13,16) & 0.03 & 0.02 & 0.003 & 0.302 \\
\hline $\mathrm{C} 24: 0$ & 0.07 & 0.08 & 0.009 & 0.725 \\
\hline C20:5 (cis-5,8,11,14,17) n3 (EPH) & 0.23 & 0.35 & 0.043 & 0.152 \\
\hline $\mathrm{C} 24: 1($ cis-15) & 0.05 & 0.07 & 0.007 & 0.146 \\
\hline C22:6 (cis-4,7,10,13,16,19) n3 (DHA) & $0.17 \mathrm{~b}$ & $0.33 \mathrm{a}$ & 0.029 & 0.001 \\
\hline$n-3$ & $1.18 \mathrm{~b}$ & $1.70 \mathrm{a}$ & 0.093 & 0.002 \\
\hline$n-6$ & 7.01 & 11.27 & 1.223 & 0.081 \\
\hline LA/ALA & 8.69 & 11.29 & 1.346 & 0.348 \\
\hline
\end{tabular}

Mean: mean value; SEM: standard error of mean; SB: soybean group; HSC: hempseed cake group; Values in each row with different letters show significant difference $(\mathrm{P}<0.05)$. 
Table 10. Antioxidative status of lambs' meat and liver

\begin{tabular}{l|rr|r|c}
\hline \multirow{2}{*}{\multicolumn{1}{c|}{ Parameter }} & \multicolumn{2}{|c|}{ Group (mean) } & \multirow{2}{*}{ SEM } & \multirow{2}{*}{ P-value } \\
\cline { 2 - 3 } & SB & HSC & & \\
\hline MDA (mg/kg liver) & 8.45 & 5.80 & 1.057 & 0.218 \\
MDA (mg/kg muscle) & 3.18 & 2.85 & 0.162 & 0.313 \\
DPPH liver (\%)* & 51.23 & 45.11 & 1.908 & 0.110 \\
DPPH muscle (\%)** & 55.13 & 55.04 & 0.532 & 0.936 \\
\hline
\end{tabular}

Mean: mean value; SEM: standard error of mean; SB: soybean group; HSC: hempseed cake group; MDA: malondialdehyde; *\% DPPH radical scavenging activity at final liver concentration $0.01 \mathrm{~g} / \mathrm{ml}$; ** \% DPPH radical scavenging activity at final meat concentration $0.02 \mathrm{~g} / \mathrm{ml}$.

Slaughter data revealed higher $(\mathrm{P}<0.05)$ carcass yield in the SB group than in the HSC group (Table 8), while the rest of the carcass traits, as well as colour indices and $\mathrm{pH}$ values $(\mathrm{P}>0.05)$ did not differ between the groups. The concentration of fatty acids in $m$. semimebranosus did not differ significantly except for DHA concentration and $n-3$ fatty acids, which were higher $(\mathrm{P}<0.05)$ in HSC group than in SB group (Table 9). Also, there was a tendency $(\mathrm{P} \leq 0.10)$ of increase in the content of $\mathrm{C} 4: 0, \mathrm{C} 12: 0$, C15:1 cis10, LA, ALA, PUFA, $n-6$ fatty acids and UFA, as well as the decrease of C16:0, C16:1 cis9, and SFA, although differences were not significant.

Neither MDA nor DPPH in muscle and liver differed significantly $(\mathrm{P}>0.05)$, although the SB group showed some increase in the content of these parameters when compared to the HSC group (Table 10).

\section{Discussion}

In the present study, there were no significant differences found in body weight, feed intake and conversion, except for daily weight gain, which was higher in HSC compared to SB group observed from days 15 to 30. In the present research, HSC inclusion in lambs' diet had no effects on carcass traits, except for dressing percentage, which was significantly lower when compared to SB group (50.68\% vs. 53.80\%, respectively). As determined in the study by Makarechian et al. (1978), it is known that postweaning average daily gain is in negative correlation with dressing percentage of lambs. Mustafa et al. (1999) did not determine changes in production traits and feed intake of lambs when soybean was replaced by hempseed meal at levels up to $20 \%$ of the dietary DM. Karlsson and Martinson (2011) did not determine significant differences in feed intake when feeding lambs with HSC supplemented diets. In their research on steers, Turner et al. (2008) studied addition of protein supplement of $0.2-1.4 \mathrm{~kg}$ HSC as-fed or $0.7 \mathrm{~kg}$ rolled barley and $0.7 \mathrm{~kg}$ of soybean meal. Results showed no differences in hot carcass weights and shear force on cooked $\mathrm{m}$. longissimus dorsi from each steer.

In the present study, no significant differences were found in the HSC group in comparison with the SB group, except for the lower MCHC content in whole blood 
on day 15. However, there was a tendency of decrease in the HGB and MCH content in HSC blood compared to SB group.

All hematological parameters were within physiological values for sheep (Kramer, 2000). In guinea pigs, Karimi et al. (2008) determined hemoglobin concentration and leukocyte number values, which showed a steady, but not significant decline $(\mathrm{P}>0.05)$. In laying hens fed diet containing hemp seed $(10,20$ or $30 \% \mathrm{DM})$ and hempseed oil (4.5 or 9\% DM), Neijat et al. (2014) did not determine significant changes in plasma level of metabolites (proteins, glucose, uric acid, cholesterol) and electrolytes ( $\mathrm{Na}, \mathrm{Ca}, \mathrm{K}, \mathrm{Cl}$ and $\mathrm{P}$ ), which is similar to the results of the present study. However, these authors determined significantly lower GGT activity in 10 and $20 \%$ hemp seed inclusion in diet or $4.5 \%$ hempseed oil, as well as similar changes of AST activity in hempseed groups. Hemp seed intake can induce significant changes in serum lipid profiles in non-ruminants (Kang and Park, 2007; Karimi and Hayathghaibi, 2006).

In comparison with lambs in SB group, the blood of lambs fed diet with HSC showed increased $\mathrm{Mg}$ concentration, which could be associated with higher content of Mg in HSC, as reported in the study by Callaway (2004). When compared to SB group, lower concentration of urea in blood of HSC lambs was probably caused by higher RUP (rumen-undegradable protein) in hempseed cake. The research by Mustafa et al. (1999) showed that hempseed meal was an excellent natural source of rumen undegraded crude protein $(774.2 \mathrm{~g} / \mathrm{kg}$ of crude protein). The research by Akhtar et al. (2017) showed the effect of varying levels of RUP in diet of early lactating cows and determined decreased $(\mathrm{P}<0.05)$ blood urea nitrogen at higher dietary RUP level. Hempseed cake in lambs' diets did not influence activity of the enzymes, like AST, ALT, ALP, GGT and CK, as well as SOD and GPx enzymes, thus not affecting the antioxidant status of lambs' blood, in comparison with SB group. Unlike in the SB group, feeding lambs with HSC led to the tendency of increased LA concentrations in lamb meat. Similar trend was determined by Turner et al. (2012) when feeding lambs with HSC diets. In this study, there was a tendency of PUFA increase determined in muscle, and this was particularly pronounced for the 18:2 n-6 and other $n-6$ desaturation and elongation products, leading to the tendency of increase of total $n-6$ fatty acids. The HSC group had significantly higher concentration of C22:6 n-3 than the SB group. In the present study, this can be related to the effect of diet (Table 1). If compared to the SB group, meat of HSC lambs had significantly higher total $n$ - 3 fatty acid concentration, while $n-6$ fatty acid concentration showed a tendency of increase.

In the research by Turner et al. (2012), HSC in diet of lambs resulted in the highest muscle C22:6 n-3 concentration. Cremonesi et al. (2018) observed that hemp seed addition in the diet caused changes in rumen biohydrogenation pathway in dairy goats by increasing the C18:2 n- 6 biohydrogenation intermediates, compared to rumen liquor of goats fed linseed that increased concentration of C18:3 isomers. The tendency of increase in the concentration of ALA in lamb meat of HSC group compared to SB in the present study could be caused by implementation of hempseed cake in feed mixture, which is rich in ALA (Table 1). Similar conclusion was reached by Mierlita (2018), when feeding sheep with $180 \mathrm{~g} /$ day hemp seeds in diets to in- 
crease concentration of ALA in ewes' milk by $66 \%$, while hempseed cake increased ALA by $49 \%$.

When compared to the SB group, analysis of parameters of oxidative status in blood and tissues of HSC lambs determined slightly increased values of blood GPX and SOD activities, as well as lower concentration of MDA and DPPH in meat and liver, although without significant differences. In the experiment of Girgih et al. (2014), SOD activity increased in plasma of hypertensive rats fed with hempseed protein hydrolysate. Palade et al. (2019) investigated antioxidant effect of hempseed diet rich in $n-6$ PUFA in sows during late gestation and lactation and their offspring. The results showed a significant improvement in the oxidative status of sows and piglets fed diets with hemp seed, since MDA was reduced in plasma and reactive oxygen species. Oxidative stability of milk was preserved when sheep were fed with diets containing hempseeds or HSC, as confirmed by higher $\alpha$-tocopherol concentration in sheep milk, higher total antioxidant capacity, and lower MDA concentration in sheep milk (Mierlita, 2018). Even though HSC exhibits the improvement of the antioxidant status determined in sheep and sows and their piglets, in the present study positive effect on antioxidant status improvement was missing in lambs' meat and liver. In the present study, the antioxidant status of lambs was preserved, and it was not under the diet influence, which requires further research with longer duration of HSC implementation in lambs' diets.

\section{Conclusion}

When considering production traits of lambs and metabolic profile of lambs' blood, as well as fatty acids in meat and antioxidative status, the present study confirmed that the inclusion of HSC up to $12 \%$ as replacement to soybean in diet of lambs is justified. Future research shall be carried out to examine higher levels of HSC supplementation in lambs' diets with longer duration of experiment in order to achieve significant improvement of fatty acid profile of lambs' meat, as well as improvement of their antioxidant status.

\section{Acknowledgements}

The study was carried out within the research team Innovative breeding and technological processes in animal production (No. 1126) at the Faculty of Agrobiotechnical Sciences Osijek.

\section{Declaration of interest statement}

No potential conflict of interest was reported by the authors.

\section{References}

A khta r M., N is a M.U., Ha y at Z., S a rw a r M. (2017). Effect of varying dietary rumen undegraded protein on nutrient intake, nutrient digestibility and production performance in early lactating crossbred cows. Pak. J. Agri. Sci., 54: 923-930.

Antunović Z., Klir Ž., Šperanda M., Cavar S., Mioč B., Novoselec J. (2017). Production traits, blood metabolic profile and fatty acids of meat and tallow in response to the 
partial replacement soybean meal with peas in organic lambs' feed. Arch. Anim. Breeding, 60: $409-417$.

Antunović Z., Klir Ž., Š peranda M., S i čaja V., Č olović D., Mi oč B., N o vos elec J. (2018). Partial replacement of soybean meal with pumpkin seed cake in lamb diets: Effects on carcass traits, haemato-chemical parameters and fatty acids in meat. S. Afr. J. Anim. Sci., 48: 695-704.

Antunović Z., Šperanda M., Mi oč B., Klir Ž., Ća va r S., N ov o s e le c J. (2019). Partial replacement of soybean meal with pea grains and sunflower cake in ewe diets: milk quality and blood biochemical parameters. R. Bras. Zootec., 48: e20180140.

AOAC (2006). Official methods of analysis of AOAC International. Association of Analytical Communities, Arlington, Virginia, USA.

Ca 11 a w a y J.C. (2004). Hempseed as a nutritional resource: an overview. Euphytica, 140: 65-72.

CIE-Commission Internationale de l'Eclairage (1976). Colorimetry: Official Recommendations of the International Commission on Illumination. Publication CIE 15: E-1.3.1. Bureau Central de la CIE, Paris, France.

Cremonesi P., Conte G., Severgnini M., Turri F., Capra E., Rapetti L., Colombini S., Chess a S., B ate 11 i G., Alves S.P., Mele M., Castiglioni B. (2018). Evaluation of the effects of different diets on microbiome diversity and the fatty acid composition of rumen liquor in dairy goats. Animal, 12: 1856-1866.

Croatian Standards Institute (2003). Milk fat - preparation of fatty acid methyl esters (ISO 15884:2002), Zagreb, Croatia

Croatian Standards Institutes (2017). Animal and vegetable fats and oils - Gas chromatography of fatty acid methyl esters - Part 2: Preparation of methyl esters of fatty acids (ISO 12966-2:2017; EN ISO 12966-2:2017), Zagreb, Croatia.

European Committee for Standardization (2006). Animal feeding stuffs - determination of amylasetreated neutral detergent fibre content (aNDF) (ISO 1647:2006; EN ISO 16472:2006). European standard, Brussels, Belgium.

European Committee for Standardization (2008). Animal feeding stuffs - determination of acid detergent fibre (ADF) and acid detergent lignin (ADL) contents (ISO 13906:2008). European standard, Brussels, Belgium.

F i s c h e r A.V., d e B o er H. (1994). The EAAP standard method of sheep carcass assessment. Carcass measurements and dissection procedures, Report of the EAAP Working Group on carcass evaluation, in cooperation with the CIHEAM Instituto Agronomico Mediterraneo of Zaragoza and the CEC Directorate General for Agriculture Brussels. Livest. Prod. Sci., 38: 149-159.

Girgih A.T., A lashi A., He R., Malom o S., A luko R.E. (2014). Preventive and treatment effects of a hemp seed (Cannabis sativa L.) meal protein hydrolysate against high blood pressure in spontaneously hypertensive rats. Eur. J. Nutr., 53: 1237-1246.

Hessle A., Eriksson M., Nadeau E., Turner T., Johansson B. (2008). Cold-pressed hempseed cake as a protein feed for growing cattle. Acta Agr. Scand. A-AN., 58: 136-145.

K a n g H.K., P a rk B.S. (2007). Effects of dietary $\gamma$-fatty acids on the fatty acid composition of pork and plasma lipids in swine. J. Food Sci. Nutr., 36: 563-568.

K a r i m i I., H a y a t g h a ib i H. (2006). Effect of Cannabis sativa L. seed (hempseed) on serum lipid and protein profiles of rat. Pak. J. Nutr., 5: 585-588.

Karimi I., Hay atgha ibi H., Yous efi J., S aberivand A., Z a vare h S. (2008). The effect of Cannabis sativa L. (hemp seed) on haematological parameters in guinea pigs. Cardiovasc. Hematol. Disord. Drug Targets, 7: 288-290.

Karls s o n L., Martin s s on K. (2011). Growth performance of lambs fed different protein supplements in barley-based diets. Livest. Sci., 138: 125-131.

Karls s on L., Fine 11 M., Martins s on K. (2010). Effects of increasing amounts of hempseed cake in the diet of dairy cows on the production and composition of milk. Animal, 4: 1854-1860.

Klir Z., Castro-Montoya J.M., Novoselec J., Molkentin J., Domacinovic M., Mioc B., Dickhoefer U., Antunovic Z. (2017). Influence of pumpkin seed cake and extruded linseed on milk production and milk fatty acid profile in Alpine goats. Animal, 11: 1772-1778.

K li r Ž., N ov o s e le c J., A n t u n ovi ć Z. (2019). An overview on the use of hemp (Cannabis sativa L.) in animal nutrition. Poljoprivreda, 25: 52-61. 
K r a m e r J.W. (2000). Normal hematology of cattle, sheep, and goats. In: Schalm's veterinary hematology, Kramer B.F., Zinkl J.G, Jain N.C., (eds), 5th ed. Baltimore, Lippincott Williams \& Wilkins, pp. 1057-1084.

Liu F., Dai R., Zhu J., Li X. (2010). Optimizing color and lipid stability of beef patties with a mixture design incorporating with tea catechins, carnosine, and $\alpha$-tocopherol. J. Food Eng., 98 : 170-177.

Makarechian M., Whiteman J.V., Walters L.E., Munson A.W. (1978). Relationships between growth rate, dressing percentage and carcass composition in lambs. J. Anim. Sci., 46: 1610-1617.

Mierlit a D. (2016). Fatty acid profile and health lipid indices in the raw milk of ewes grazing parttime and hemp seed supplementation of lactating ewes. S. Afr. J. Anim. Sci., 46: 237-246.

Mi e r lit a D. (2018). Effects of diets containing hemp seeds or hemp cake on fatty acid composition and oxidative stability of sheep milk. S. Afr. J. Anim. Sci., 48: 504-515.

Must a fa A.F., Mc K innon J.J., Christensen D.A. (1999). The nutritive value of hemp meal for ruminants. Can. J. Anim. Sci., 79: 91-95.

National Research Council (2007). Nutrient requirements of small ruminants: sheep, goats, cervids, and new world camelids. The National Academies Press, Washington, DC, USA.

Ne ij at M., Gakhar N., N e ufeld J., Hous e J.D. (2014). Performance, egg quality, and blood plasma chemistry of laying hens fed hempseed and hempseed oil. Poultry Sci., 93: 2827-2840.

P a l a d e L.M., H ab e a nu M., Marin D.E., Che d e a V.S., P i s tol G.C., Gros u I.A., G h e or g h e A., R o p o t a M., Ta r a n u I. (2019). Effect of dietary hemp seed on oxidative status in sows during late gestation and lactation and their offspring. Animals, 9: 194.

Qwele K., Hug o A., O y e d e mi S.O., Moy o B., Ma si ka P.J., Muchenje V. (2013). Chemical composition, fatty acid content and antioxidant potential of meat from goats supplemented with Moringa (Moringa oleifera) leaves, sunflower cake and grass hay. Meat Sci., 93: 455-62.

R u s s e 1 A. (1991). Body condition scoring of sheep. In: Sheep and goat practice, Boden E. (ed.). Bailliere Tindall, Philadelphia, USA, pp. 3.

R u s s o R., R e g g i a n R R. (2013). Variability in antinutritional compounds in hempseed meal of Italian and French varieties. Plant, 1: 25-29.

Sniffen C.J., O' Connor D.J., Van Soest P.J., Fox D.G., R us s ell J.B. (1992). A net carbohydrate and protein system for evaluating cattle diets: Carbohydrate and protein availability. J. Anim. Sci., 70: 3562-3577.

Turner T., Hess le A., Lund strom K., P i ckova J. (2008). Influence of hempseed cake and soybean meal on lipid fractions in bovine M. longissimus dorsi. J. Acta Agr. Scan., Section A-Anim. Sci., 58: 152-160.

Turner T.D., K a rls s o n L., M a pi y e C., Rolland D.C., Martins son K., Dugan M.E.R. (2012). Dietary influence on the $\mathrm{m}$. longissimus dorsi fatty acid composition of lambs in relation to protein source. Meat Sci., 91: 472-477.

W a n g Q., X i o n g Y.K. (2019). Processing, nutrition, and functionality of hempseed protein: a review. Compr. Rev. Food Sci. F., 18: 936-952.

Received: 9 III 2020

Accepted: 15 X 2020 